\title{
Robotic pancreatoduodenectomy for large solid pseudopapillary tumor of the pancreas using precise and systematic vascular control technique
}

\author{
Raja KALAYARASAN*
}

Department of Surgical Gastroenterology, JIPMER, Pondicherry, India

Introduction: Large tumors in the head of the pancreas pose technical challenges during resection in minimally invasive pancreatoduodenectomy (PD). The precise and systematic vascular control of the pancreaticoduodenal branches of the superior mesenteric artery and vein during robotic PD for a large solid pseudopapillary tumor of the pancreas is described in this video.

Methods: A 16-year-old female presented with a lump in the abdomen, early satiety, and epigastric pain of 3 months duration. Contrast-enhanced computed tomography abdomen revealed $7.1 \times 6.2 \mathrm{~cm}$ solid mass with cystic areas in the head of the pancreas. The tumor was compressing the portal vein with dilated pancreaticoduodenal veins. In the present technique, the anterosuperior pancreaticoduodenal vein is controlled first, followed by the superior pancreaticoduodenal artery (by ligating Gastroduodenal artery). This is followed by the control of inferior pancreaticoduodenal veins and arteries. The posterosuperior pancreaticoduodenal vein is ligated at the end to prevent vascular congestion of the uncinate region.

Results: The duration of surgery and intraoperative blood loss were 440 minutes and $200 \mathrm{~mL}$, respectively. The patient had an uneventful postoperative course and discharged on the sixth postoperative day. Histopathological examination confirmed the diagnosis of the solid pseudopapillary tumor. At nine months follow-up, the patient remains asymptomatic with no recurrence.

Conclusions: A stepwise precise vascular control described in this report could minimize intraoperative bleeding during robotic PD for large tumors in the head of the pancreas. 